\title{
USING COMPUTATIONAL GEOMETRY TO IMPROVE PROCESS RESCHEDULING ON ROUND-BASED PARALLEL APPLICATIONS
}

\author{
RODRIGO DA ROSA RIGHI, VLADIMIR MAGALHÃES GUERREIRO, GUSTAVO ROSTIROLLA, VINICIUS FACCO \\ RODRIGUES, CRISTIANO ANDRÉ DA COSTA AND LEONARDO DAGNINO CHIWIACOWSKY *
}

\begin{abstract}
Process rescheduling is a known technique to face with system heterogeneity and dynamism, being especially pertinent on Bulk Synchronous Parallel (BSP) programs. These programs are organized in a set of round-based supersteps, in which the slowest process determines the moment of synchronization. This approach motivated us to develop a first model called MigBSP, which combines computation, communication and migration costs metrics for process rescheduling decisions. MigBSP originally employed an heuristic that could select either a single or a collection of process to migrate at each load balancing invocation. The first proposal is not reactive, so you should manually setup a percentage of processes to be migrated as input parameter for the load balancing model. In this work, two novel heuristics, named MigCube and MigHull, are proposed to choose the candidate processes for migration and their destination. Both heuristics consider the use of computational geometry for plotting computation, communication and migration costs metrics in a 3D graph, so both 'which' and 'where' load balancing questions can be answered without any user intervention. We believe that the contribution is not only in the MigBSP landscape, but also for the BSP community, who is trying to enhance performance in round-based applications in an effortless way. In addition to the description of MigCube and MigHull, this article also presents their evaluations with performance gains of up to $42 \%$ when enabling process migration over a subset of the Grid5000 infrastructure.
\end{abstract}

Key words: Computational Geometry, Process Migration, Performance, Dynamism, Grid Computing

AMS subject classifications. 15A15, 15A09, 15A23

1. Introduction. Process migration is a useful mechanism to offer runtime load balancing, mainly in dynamic, complex and heterogeneous environments. Generally, process migration requires explicit rescheduling calls within the application [11]. A different migration approach happens at middleware level, where changes in the application code and previous knowledge about the system are usually not required. Considering this, we have developed a process rescheduling model for grid computing architectures called MigBSP [28]. We decided to work with round-based applications, such as those that follow the BSP (Bulk-Synchronous Parallel) programming model [33]. Concerning the choose of migration processes, MigBSP creates a priority list based on the highest Potential of Migration (PM) of each process. PM is a decision function that combines the migration costs with data from computation and communication phases in order to create a unified scheduling metric.

Taking profit from the highest PM of each process, MigBSP could originally employ one of two methods to select the candidate processes for migration. As illustrated in Figure 1.1, MigBSP can select one or a group of processes located on the top of the list. The second case is viable thanks to a predefined percentage that acts over the highest PM value. Although we achieved good results particularly with this second approach [28], we agree that the use of another percentage value could eventually determine better migration results. Consequently, a question arises: Using the PM idea, how can one reach an optimized percentage of migratable candidates on dynamic environments? A solution involves the testing of several hand-tuned parameters for each new BSP application and a comparison among the results.

After developing the first version of MigBSP, we focused our research on investigating new heuristics and metaheuristics in order to fill the aforementioned gap. We followed this rationally because both scheduling and rescheduling techniques are classified as NP-hard problems [15]. Taking into account metaheuristics, Genetic Algorithms [14, 22, 26], Simulated Annealing [13, 34], Artificial Bee Algorithms [16, 3], Pareto Search [32] and Hybrid Schemes [18] are commonly used for these tasks. Considering their iterative nature, they are known by reaching high-quality solutions meanwhile paying a high-computational time for achieving optimal or nearoptimal solutions. On the other hand, heuristics are faster than metaheuristics, since they operate with mental shortcuts to ease the cognitive load of making a decision [9]. Thus, heuristics such as min-min and max-min operate by trading optimistically, completeness, accuracy, or precision for speed. When analyzing the state-ofthe-art on migration-aware BSP communication libraries $[8,19,21,24,25,28$, 35], we still observe that both

*Applied Computing Graduate Program, Universidade do Vale do Rio dos Sinos, São Leopoldo - Rio Grande do Sul - Postal Code 93022-000 - Brazil (rrrighi@unisinos.br). 


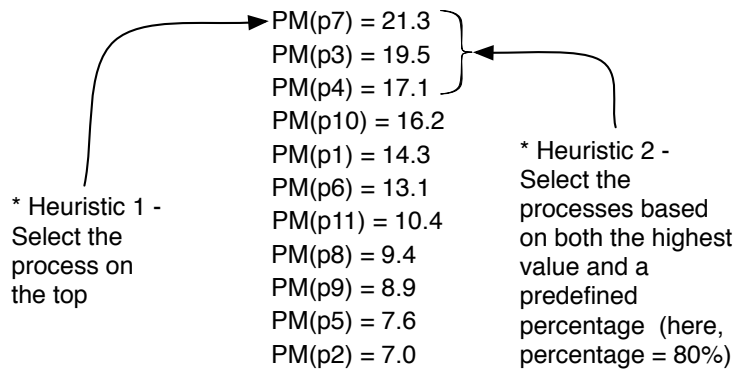

Fig. 1.1: Current MigBSP's methods for choosing the candidate processes for migration based on a decision function named Potential of Migration (PM).

heuristics and metaheuristics techniques are not employed to offer rescheduling under the following constraints: (i) combination of multiple metrics; (ii) automatic selection of candidate processes for migration without user intervention.

When process (re)scheduling is considered, two timers are involved: calculus complexity and quality of the mapping. Both measures are used in heuristics for optimizing the MigBSP's initial approaches. In this regard, we developed two novel heuristics named MigCube and MigHull for automatically selecting one or more candidates for migration at each rescheduling attempt. They solve a 3D geometric query problem taking profit from the computation, communication and migration costs metrics of the PM as the values for the $\mathrm{x}$, $\mathrm{y}$ and $\mathrm{z}$ axes. So, the scientific contribution of the article consists in exploring computational geometry concepts to select the most suitable points arranged in a three-dimensional space, consequently indicating the processes for migration, without needing any intervention when considering the user viewpoint. MigCube explores the Euclidean distance [12] among the points while MigHull extends the idea of Convex Hull for a 3D setting [4].

This article presents the algorithms of MigCube and MigHull in detail, followed by their evaluation when using two BSP scientific applications over a subset of the Grid5000 infrastructure ${ }^{1}$. Besides not needing a particular parameter in the model at compilation time, the results also show the benefits of selecting a more appropriate number of migratable processes instead of selecting just one or a percentage of them. The contribution of both proposed heuristics does not appear only in the MigBSP scope, but also for the BSP community who is interested in efficient migration process at middleware level in an effortless way.

The remainder of this article will first introduce the fundamental concepts in Section 2, explaining how MigBSP works in detail. The main part of the paper belongs to Section 3, where both MigCube and MigHull algorithms are proposed. Sections 4 and 5 show the employed methodology and the results, respectively. Related work is discussed in Section 6. Finally, Section 7 emphasizes the scientific contribution of the work and notes challenges that we can address in the future.

2. Fundamental Concepts. This section explains the functioning of MigBSP, emphasizing its rationales and parameters. MigBSP is a rescheduling model that works over heterogeneous resources, joining the power of clusters, supercomputers and local networks. The heterogeneity issue considers the processor's clock (all processors have the same set of instructions), as well as the network bandwidth. Such an architecture is assembled with Sets (sites or clusters) and Set Managers. Set Managers are responsible for scheduling, capturing data from a Set and exchanging it among other managers [28].

The decision for process remapping is taken at the end of a superstep. A BSP program has an arbitrary number of supersteps, each one composed by a local computation phase on each process, a global and arbitrary communication phase among the processes and a synchronization barrier [33]. Aiming at not trying to test process rescheduling at each conclusion of superstep, we designed a parameter named $\alpha$ to control the interval of supersteps between two consecutive attempts for process rescheduling. Thus, we applied two adaptations

\footnotetext{
${ }^{1}$ https: //www.grid5000.fr/
} 
that control the value of the $\alpha(\alpha \in \mathbb{N})$ in order to reduce the scheduling model intrusiveness: (i) to postpone the rescheduling call if the processes are balanced or to turn it more frequent, otherwise; (ii) to delay this call if a pattern without migrations on $\omega$ past calls is observed. Thus, $\alpha$ is automatically updated at each rescheduling call and will indicate the interval for the next one (more details in [28]). A shorter initial value of $\alpha$ will bring better reactivity on application reorganization, since process rescheduling will be evaluated as soon reaching the $\alpha^{t h}$ superstep. So, this configuration implies on reconfiguring the application sooner, benefiting the remaining of the execution with an optimized process-resources mapping. However, if process migration is inviable (due to the large number of bytes to be transferred or a prohibitive network latency overhead, for example), a shorter $\alpha$ will cause more overhead in the normal execution of the application. In this last case, process rescheduling is tested more frequently, but no migrations take place actually.

The answer for 'Which' is solved through our decision function called Potential of Migration (PM). Each process $i$ computes $n$ functions $P M(i, j)$, where $n$ is the number of Sets and $j$ means a particular Set. The key rationale consists in performing only a subset of the processes-resources tests at the rescheduling moment. The value of $P M(i, j)$ is found using Computation, Communication and Memory metrics as presented in Equations 2.1-2.4. A previous paper describes them in detail [28]. The greater the value of $P M(i, j)$, the more prone the processes will be to migrate.

$$
\begin{aligned}
\operatorname{Comp}(i, j) & =P_{\text {comp }}(i) \times C T P_{k+\alpha-1}(i) \times \operatorname{ISet}_{k+\alpha-1}(j) ; \\
\operatorname{Comm}(i, j) & =P_{\text {comm }}(i, j) \times B T P_{k+\alpha-1}(i, j) \\
\operatorname{Mem}(i, j) & =M(i) \times T(i, j)+\operatorname{Mig}(i, j) ; \\
P M(i, j) & =\operatorname{Comp}(i, j)+\operatorname{Comm}(i, j)-\operatorname{Mem}(i, j) .
\end{aligned}
$$

Computation metric $\operatorname{Comp}(i, j)$ considers a Computation Pattern Pcomp $(i)$ that measures the stability of a process $i$ regarding the number of instructions at each superstep. This value is close to 1 if the process is regular and close to 0 otherwise. Furthermore, we also have a computation time prediction $C T P(i)$ for process $i$ based on all computation phases between two rescheduling activation. In this way, here $k$ refers to the index of the last call for process rescheduling and $k+\alpha-1$ means the interval of supersteps from the last to the current rescheduling attempt. The metric $\operatorname{Comp}(i, j)$ also presents an index $I S e t(j)$ which informs the average computation capacity of Set $j$. In the same way, Communication metric $\operatorname{Comm}(i, j)$ computes the Communication Pattern Pcomm $(i, j)$ between processes and Sets. Furthermore, this metric uses communication time prediction $B T P(i, j)$ considering data between two re-balancing activation. Comm $(i, j)$ increases if process $i$ has a regular communication with processes from Set $j$ and performs slower communication actions to this Set. The metric $\operatorname{Mem}(i, j)$ considers process memory $M(i)$, transferring rate $T(i, j)$ between considered process $i$ and the manager of target Set $j$, as well as migration costs $M i g(i, j)$. These costs are dependent of the operating system, as well as the migration tool [28].

At each rescheduling call, each process passes its highest $P M(i, j)$ to its Set Manager. This last entity exchanges the PM of the processes with other managers. As described earlier, each manager creates a decreasingsorted list and selects either the process on the top or a percentage of them for testing the migration viability. Here, besides using the abstraction of Set, this test also considers the following data: (i) the external load on source and destination processors; (ii) the processes that both processors are executing; (iii) the simulation of considered process running on a destination processor; (iv) the time of communication actions considering local and destination processors; (v) migration costs. We used these five information to compute the migration viability of each process through a relationship between two timers: $t 1$ and $t 2 . t 1$ means the superstep time of process $i$ in the current processor, while $t 2$ encompasses its execution on the other processor and it includes the migration costs. Process migration takes place if $t 1>t 2$.

3. MigCube and MigHull: Proposal of Novel Heuristics to Select the Candidates for Migration. This article proposes MigCube and MigHull heuristics to improve efficiency on selecting process of BSP 
applications at the rescheduling moment. The main idea is to outperform the current MigBSP strategies for this task, particularly without user intervention at editing or launching time to set model parameter for selection purposes. At the application perspective, the use of a particular selection policy or even process rescheduling facility is totally hidden from the user. Usually, for the submission of a BSP application in a grid, it is previously compiled with a rescheduling-aware BSP library, informing an initial processes-nodes scheduling [38]. Figure 3.1 depicts the software stack when using MigBSP. The gray boxes represent the scope of this article.

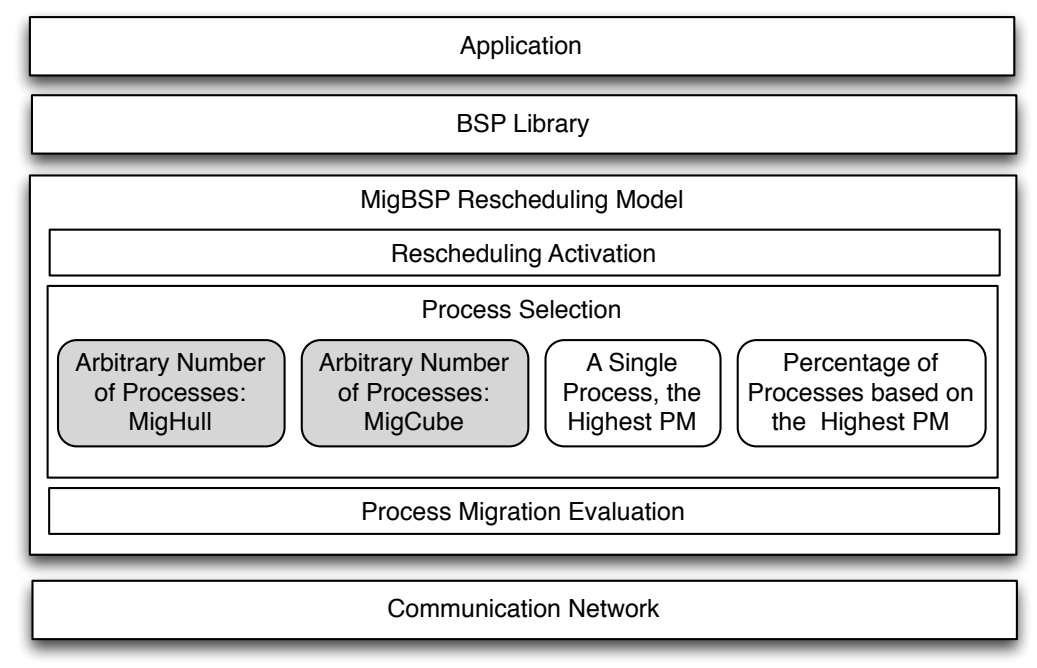

Fig. 3.1: Software stack when using the novel heuristics for BSP process rescheduling

BSP applications have their performance always driven by the slowest process, so both heuristics try to optimize the number and the selection of processes to eventually migrate so that the remaining supersteps may run faster. Unlike previous approaches, MigCube and MigHull select an arbitrary number of processes but also considering the list of the highest PM of each process. Figure 3.2 illustrates the rescheduling in a BSP application. The mapping quality of MigCube or MigHull will impact the next value of $\alpha$ parameter. If the system is classified as balanced, the value of $\alpha$ is increased in order to postpone the next call for process rescheduling.

Both MigCube and MigHull take profit from the list of the highest PM of each process. Considering that each PM identifies a process and a target Set, and since all the three assumed metrics are expressed in the same data unit, we may plot them as a single point in a 3D setting. In this way, the proposed heuristics must answer the following answer: Which points should be selected at each rescheduling call? To accomplish this, MigCube and MigHull use computational geometry to analyze a set of points, in order to efficiently find which points are close to the input query. At model level, each Set Manager compute the selected points locally, each one referring to a particular process $i$ that presents a $P M(i, j)$. After that, only the source and the target Sets (represented by $j$ in the $P M$ notation) are involved to transfer a process $i$ actually. The destination Set informs the source Set about which is the most suitable processor under its responsibility to receive the process.

Heuristic methods are employed because they are generally used to find a solution of a specific-domain problem without exhaustively searching the entire solution space [36]. Thus, such algorithms can usually achieve good solutions in a small computational time. This is special pertinent on our case, since we have to pay the overhead inherent to the migration process. Nevertheless, we could use metaheuristic methods rather than heuristic ones. However, metaheuristics represent more general approximate algorithms which are defined as upper level techniques that guide strategies underlying heuristics to solve specific optimization problems [31]. For this reason, metaheuristics sometimes require high processing time to attain near-optimal solutions, especially for large-size problems. Anyway, both single solution-based or population-based metaheuristics could be used, 


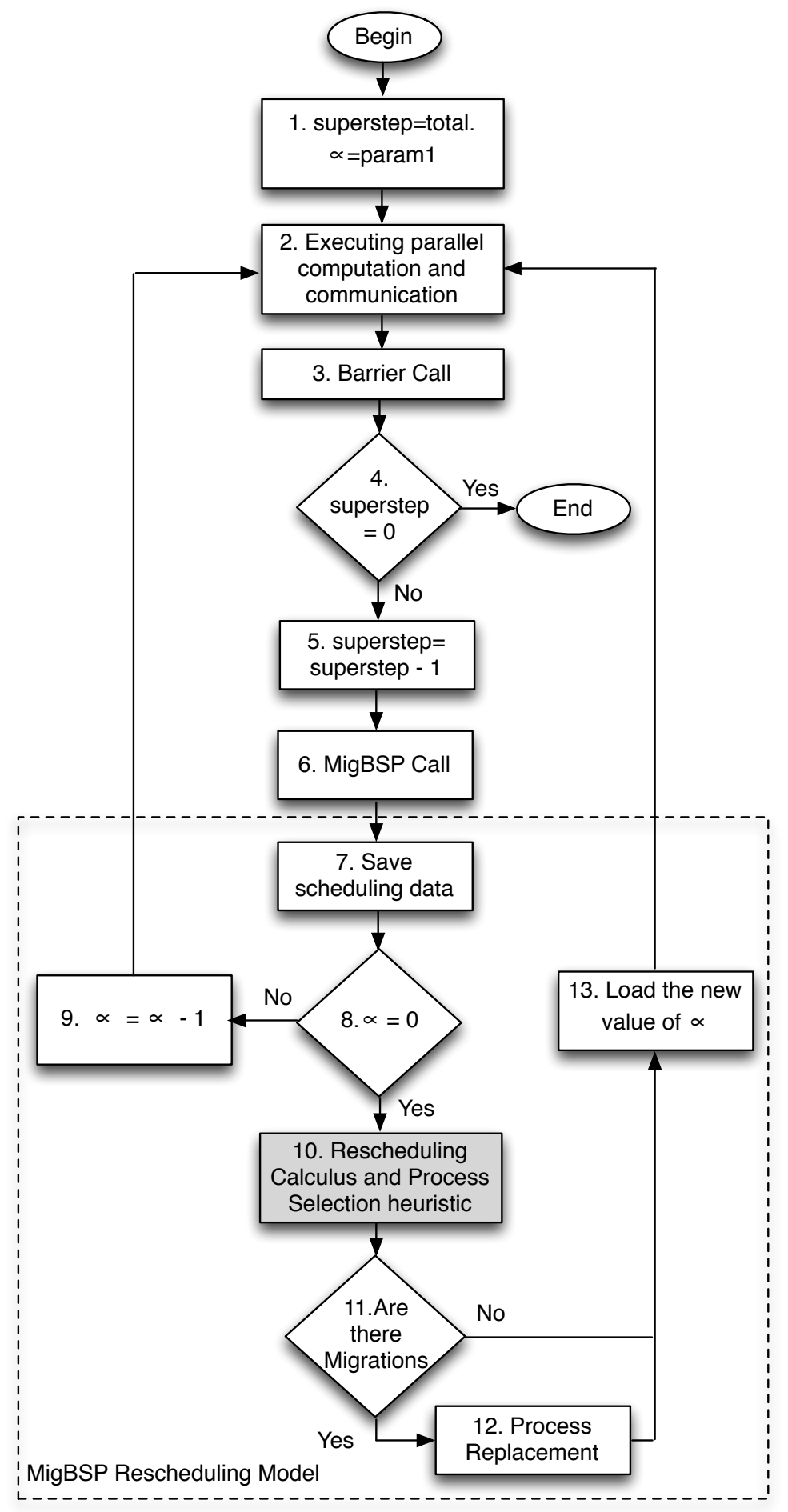

Fig. 3.2: MigBSP flowchart, where the gray box represents the work of MigCube or MigHull. 


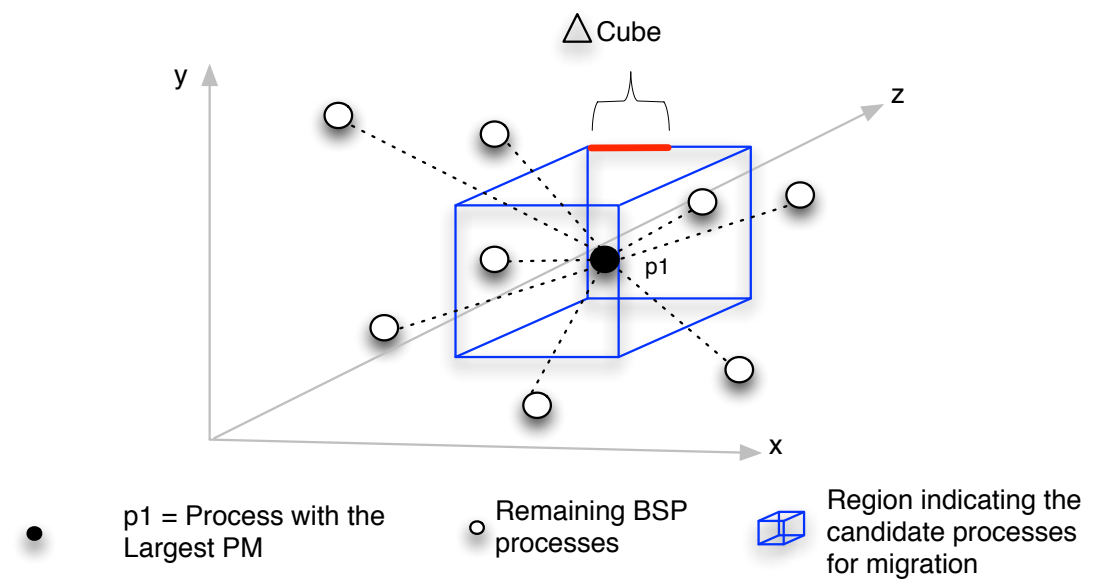

Fig. 3.3: Processes and cube representation in MigCube heuristic. Those processes that are located inside the cube will be appointed as candidate processes for migration.

such as Simulated Annealing, Tabu Search, Genetic Algorithms and Swarm Intelligence Schemes, needing only a given quality measurement to be performed.

3.1. Terminologies. Both MigCube and MigHull plot each process $p_{i}(i=1,2, \ldots, n)$ as a point in the 3D Cartesian coordinate system where $x_{i}, y_{i}$ and $z_{i}$ represent the coordinates of $p_{i}$ on each of the graph axes. Here, $x_{i}, y_{i}$ and $z_{i}$ also represent respectively, the computation, communication and memory metrics from the largest PM of $p_{i}$. In addition, a process $p_{i}$ can be also represented as $p_{i}=\left(x_{i}, y_{i}, z_{i}\right)$.

3.2. MigCube Heuristic. MigCube uses the processes' location to create a cube, so selecting as candidates those processes inside it. The algorithm starts by selecting the central point of the cube, which refers to the point that has the largest PM. Its notation is $p_{1}$ and it represents the best candidate for migration. After that, parameter $\triangle_{\text {cube }}$ is computed in accordance with Equation 3.1 as an average of the distances from the aforementioned point to the others. Equation 3.2 computes the distance between $p_{1}$ and any point $p_{i}$ in the $3 \mathrm{D}$ coordinate system. Finally, $\triangle_{\text {cube }}$ is used to situate the cube edges as defined in Equation 3.3.

$$
\begin{gathered}
\triangle_{\text {cube }}=\frac{1}{n-1} \sum_{i=2}^{n} D\left(p_{1}, p_{i}\right) ; \\
D\left(p_{1}, p_{i}\right)=\sqrt[2]{\left(x_{1}-x_{i}\right)^{2}+\left(y_{1}-y_{i}\right)^{2}+\left(z_{1}-z_{i}\right)^{2}} ; \\
\text { edge }=2 \triangle_{\text {cube }} .
\end{gathered}
$$

Figure 3.3 depicts an example of the points and the cube, where $p_{1}$ has the largest PM. Algorithm 1 presents the pseudocode of MigCube heuristic for selecting the candidate process for migration. As mentioned earlier, the idea is to select as candidates all processes inside the cube. MigCube will always select at least one process, the one with the largest PM. After the heuristic sets which processes could migrate, the model follows its normal processing, migrating or not the processes in accordance with the destination Set of each candidate process. Each process $p_{i}$ has a $P M(i, j)$ where $i$ means the process index and $j$ a target Set. So, the Manager of $j-t h$ Set is asked about a resource and migration viability is computed as explained in Section 2 (more details in [28]). 


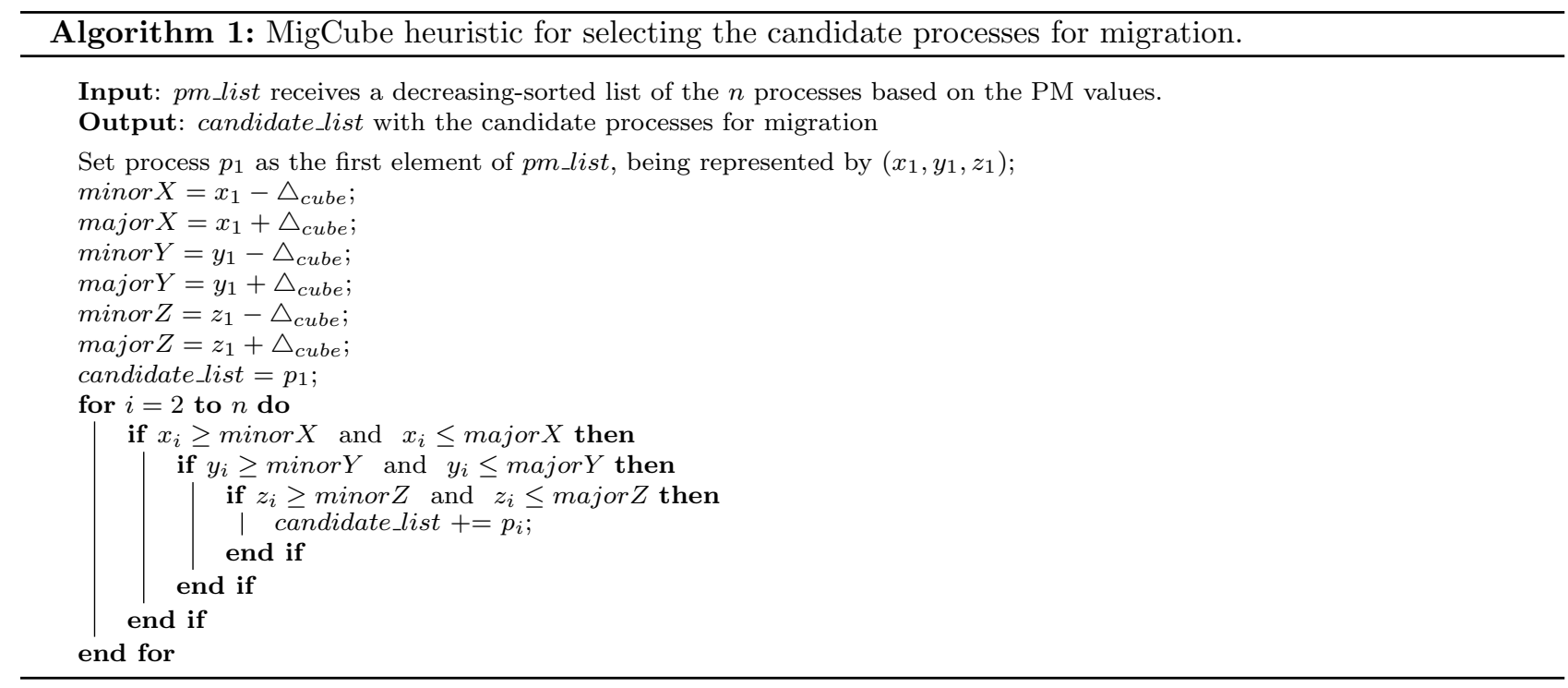

3.3. MigHull Heuristic. MigHull heuristic is a Convex Hull adaptation. In brief, the Convex Hull or Convex Envelope of a set $S$ of points in the Euclidean plane is the smallest convex set that contains $S$ [6, 4]. It can be seen as a convex polygon whose vertices are some of the points in the input set. MigHull employs the Convex Hull ideas, but providing two adaptations: (i) three-dimensional space is split in three two-dimensional planes; (ii) despite of selecting all processes, MigHull chooses only a part of them based on the two processes with the highest PM values.

We are calculating three 2D hulls, considering a pair of coordinates of each point $i$ at a time, as follows: (i) $x_{i}$ and $y_{i}$; (ii) $x_{i}$ and $z_{i}$; and (iii) $y_{i}$ and $z_{i}$. Figure 3.4 (a) illustrates this idea. Here, each process that is inside each plane concomitantly is then selected as a candidate for migration. For the standard $2 \mathrm{D}$ Convex Hull, the problem consists of finding the smallest convex polyhedron/polygon containing all the points. Thus, the native Convex Hull always selects all the points, which would not make sense for migration decision-making. In this way, we are adapting the QuickHull algorithm [5] to select processes. By default, QuickHull finds the points with the minimum and maximum $x$ coordinates and creates a line between them. The next step in the QuickHull algorithm is the selection of the point with the maximum distance from the aforesaid line, so the two points found before along with this one form a triangle. The points lying inside of that triangle cannot be part of the convex hull and can therefore be ignored in the next steps. Discarding the tested point and the previous ignored ones, the algorithm selects the next point with the maximum distance from the line and proceeds the same calculus again.

MigHull changes QuickHull as follows. Considering the plane $a-b$, where $a$ means the abscissa and $b$ the ordinate, we are considering the $a$-coordinate of the two points with the highest PM to draw a line segment between them. After that, we calculate $\triangle_{H u l l}$ as the maximum distance of coverage from this line segment to the other processes, so the processes inside this region are candidates to migrate in the scope of $a-b$ plane. Figure 3.4 (b) illustrates an example of this procedure for the $x-y$ plane, but the same is evaluated for other two planes: $x-z$ and $y-z$. In other words, by substituting $x-y$ plane by $x-z$ and $y-z$ the distance $\triangle_{H u l l}$ is also calculated in the $x-z$ and $y-z$ planes, respectively. Finally, the processes that appear as candidates concomitantly in the $x-y, x-z$ and $y-z$ planes are selected as final candidates to migrate according to the MigHull algorithm.

$$
\triangle_{H u l l}=\sigma(x, y)=\operatorname{Max}(\sigma(x), \sigma(y))
$$

Equation 3.4 shows how we are computing $\triangle_{H u l l}$ for the $x-y$ plane. Each plane has its own value for this metric. In this equation, $\sigma(a)$ for a specific axis $a$ is the standard deviation of all points (i.e. processes) when 


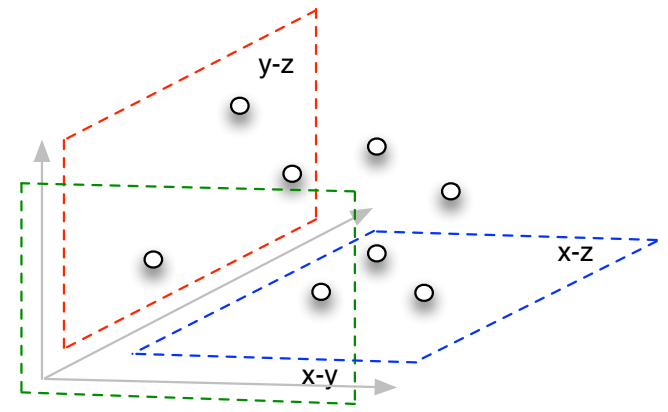

○ BSP Processes

(a)

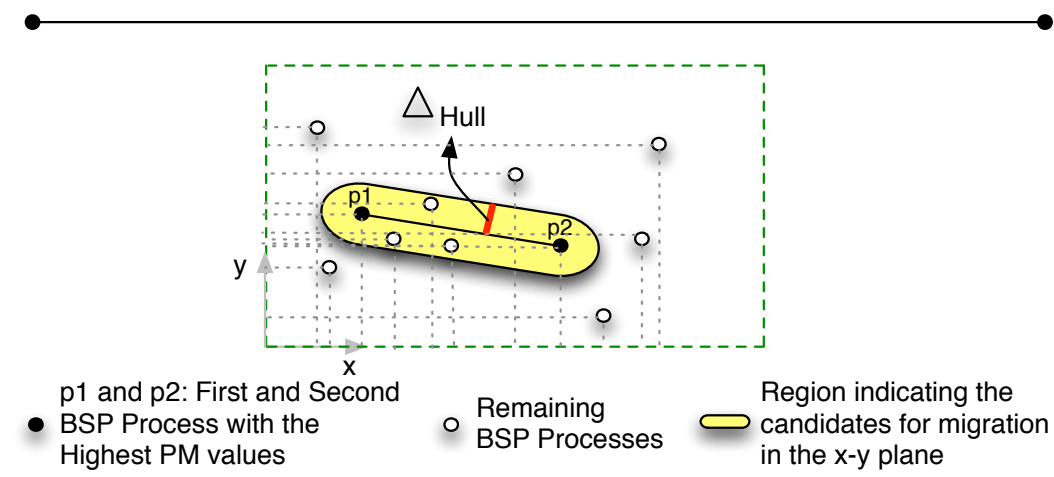

(b)

Fig. 3.4: Selection of candidate process for migration with MigHull: (a) Creating three planes $(x-y, x-z$ and $y-z)$ from the three-dimensional space; (b) partially selecting the candidate process in the $x-y$ plane. Those processes that appear concomitantly in the yellow region of the three planes are chosen for the next rescheduling step: the tests of migration viability.

considering the coordinate $a$. Using Figure 3.4 (b) as an example, we firstly take the value of the coordinate $x$ of all the 11 points, computing the standard deviation $\sigma(x)$ of these respective values. The same calculation is performed with respect to the $y$ axis, so the greatest standard deviation is selected as $\triangle_{H u l l}$ for the $x-y$ plane. In order to identify the candidate processes for migration, the distance of any point $p_{m}$ to the line determined by the points $p_{1}$ and $p_{2}$ over a specific plane (see Algorithm 2) is computed and denoted as $d\left(p_{m}, p_{1}, p_{2}, p l a n e\right.$ ). These last two points represent the processes with the highest PM. If $x$-coordinate of $p_{m}$ is lower or greater than the $x$-coordinate of points used as limits of the line segment, we are computing the Euclidean distance given by the Pythagorean formula [12]. Otherwise, we are using the perpendicular distance from a point to a line determined by $p_{1}$ and $p_{2}$. Although Algorithm 2 was developed for the $x-y$ plane, its use for $x-z$ and $y$ - $z$ is trivial and not explained here.

Figure 3.4 (b) depicts the MigHull ideas to create a region of candidate processes for migration. Contrary to MigCube, MigHull always selects at least two processes as candidates for migration: $p_{1}$ and $p_{2}$. Independently of the evaluated plane, these points always have the largest PM, so being always selected according to the MigHull algorithm. Algorithm 3 shows all steps to compute MigHull, where the processes that appear as candidates concomitantly in the $x-y, x-z$ and $y-z$ are candidates to be rescheduled. After MigHull presents the candidates, MigBSP continues its normal execution investigating the migration feasibility for each candidate through an interaction between the source and target Set Managers. MigBSP was presented in Section 2 and detailed in [28]. 

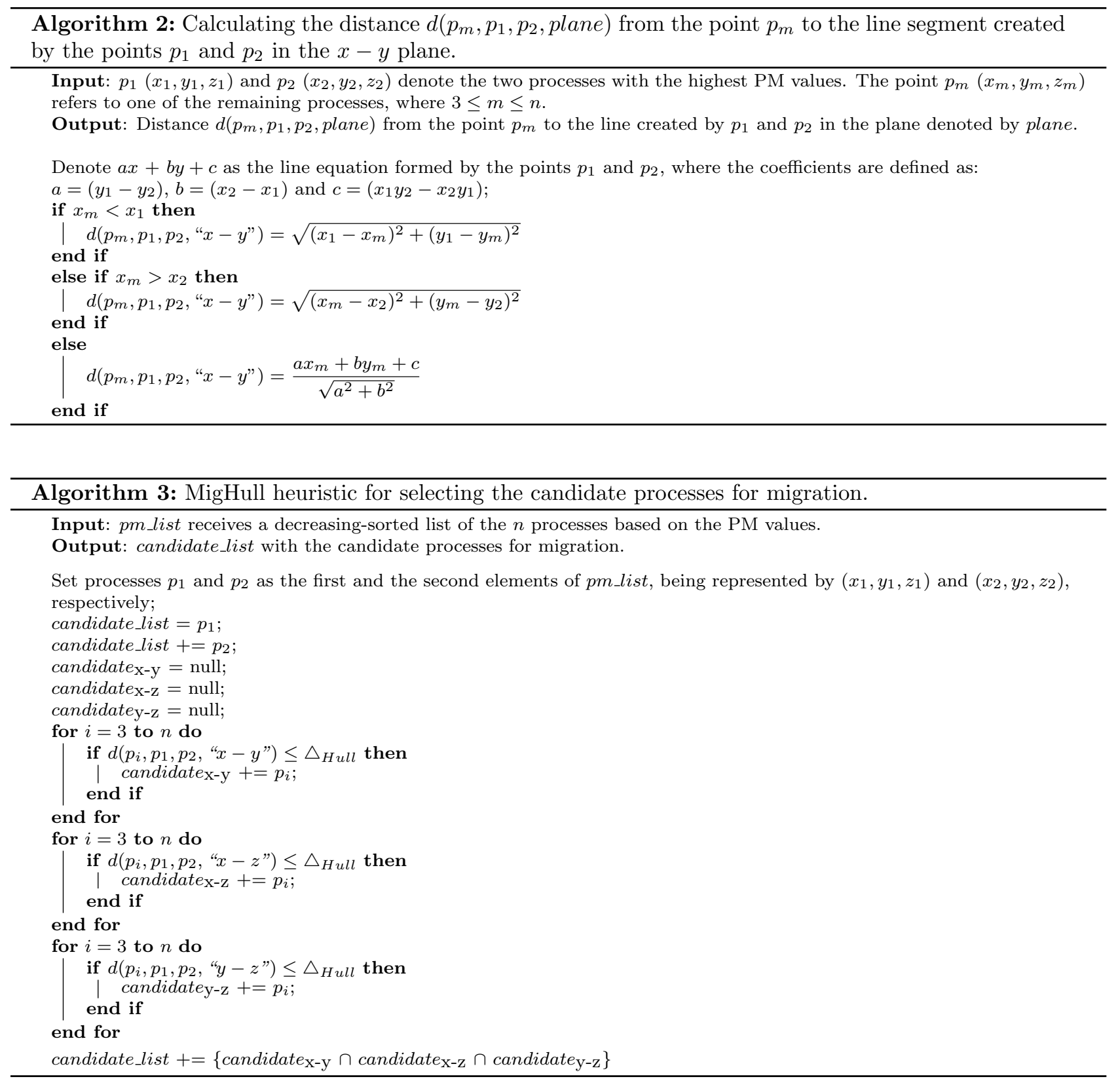

4. Evaluation Methodology. This section describes the evaluation methodology, presenting data about the evaluation technique, MigBSP parameters, execution environment and tested application. Firstly, we are using the SimGrid [2] simulator to assembly a grid computing infrastructure, because of it offers a framework to evaluate message-passing applications with different scheduling algorithms and execution platforms. We did not developed any extension to SimGrid, but only applications that use its native API (Application Program Interface). Considering that SimGrid is deterministic, a single execution of each set of parameters was done. Moreover, the number of supersteps is variable, as follows: 20, 40,60, 80 and 100. The initial value of $\alpha$ is selected among three numbers: 4, 8 and 16. We selected them because these values were used when evaluating the first version of MigBSP [28], where significant impacts on performance and overhead were perceived when changing from one value to another. Furthermore, as will be discussed in Subsection 5.4, we will present a 


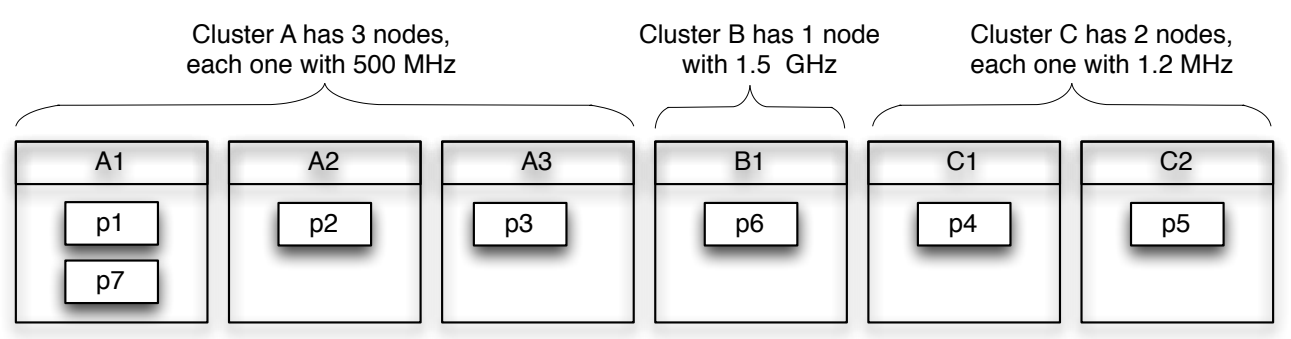

(a) Crescent

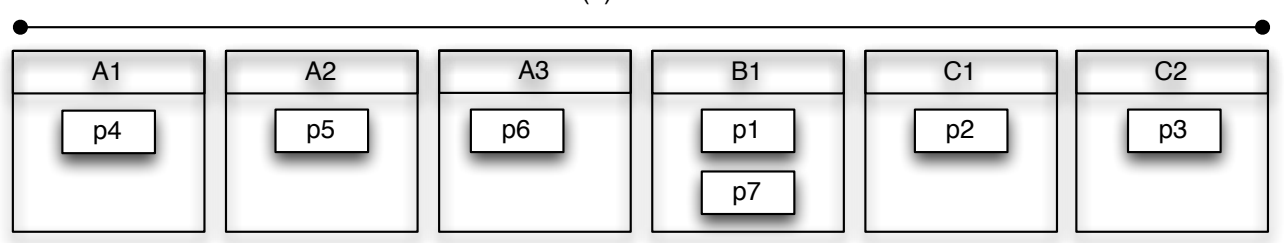

(b) Decrescent

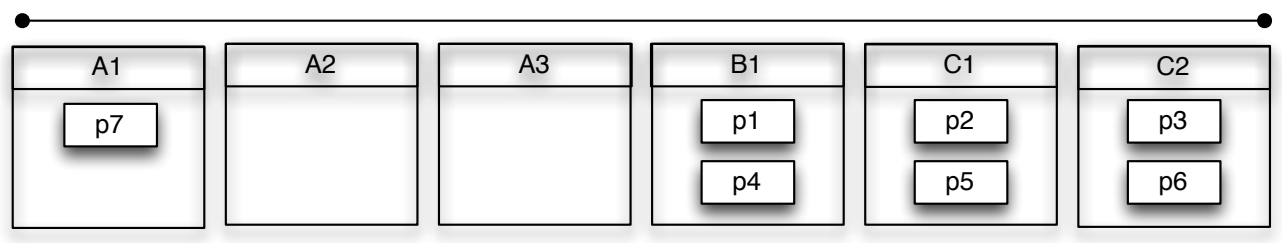

(c) CPU

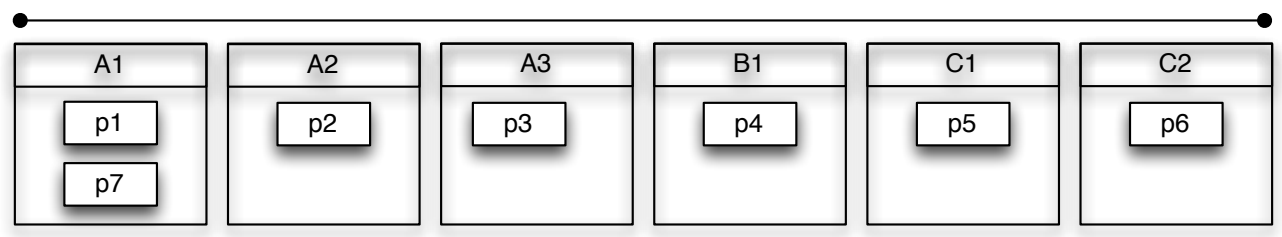

(d) Round-Robin

Fig. 4.1: Example of the four initial processes-resources scheduling employed in the tests using a hypothetical grid infrastrucuture.

comparison study among MigCube, MigHull and the originals heuristics of MigBSP, so we can analyze the impact of these values of $\alpha$ on different algorithms for process migration.

Since the MigBSP was designed for grid environments, we are testing it with the proposed heuristics over the Grid5000 platform ${ }^{2}$. In fact, this platform is an XML file used by SimGrid, denoting machines, clusters and network configurations. Besides the platform file, SimGrid also receives as input another XML file informing the first scheduling (deployment). Particularly, we are using 45 nodes, distributed in 3 distinct sites, each one offering here a single cluster. We are using the 10 nodes from cluster Chicon, 15 from cluster Capricorne and 15 nodes from cluster Suno. The hardware information is described as follow: (i) Chicon has AMD Opteron $2.6 \mathrm{GHz}$ processors with $4 \mathrm{~GB}$ of memory and a Gigabit Ethernet card; (ii) Capricorne has AMD Opteron 2.0 GHz processors, with $2 \mathrm{~GB}$ of memory and a Myrinet network card; (iii) Suno has Intel Xeon E5520 2.26GHz processors with $32 \mathrm{~GB}$ of memory and 2 Gigabit Ethernet cards. Considering the deployment file, we are working with 60 processes that are launched in accordance with an initial process-scheduling mapping. We are considering four of them, explained below and detailed in the example of Figure 4.1:

(a) Ascending: Processes are scheduled cyclically in Ascending order of nodes' processing power;

(b) Descending: Same idea of the Ascending mapping, but in reverse order, where the nodes with the higher

\footnotetext{
${ }^{2} \mathrm{http}: / /$ lists.gforge.inria.fr
} 
capacities are the first to receive processes;

(c) CPU: This mapping allocates each process to the resource that has the largest amount of free processing power on that moment;

(d) Round-Robin: It allocates the processes cyclically without taking into account any characteristics of the resources.

The initial mapping will influence the execution time directly, also influencing the rescheduling model in the same way. For example, the CPU mapping implies on using load balancing in accordance with the CPU power of the nodes, so this idea of equilibrium from the beginning tends to reduce the number of migrations at runtime. On the other hand, for example, the scheduling of all process in a single node could compromise the performance, imposing more rescheduling actions afterwards to spread them in the resource pool. Besides the initial mappings and MigBSP parameters, the tests also consider three scenarios: (i) execution of the native application, without MigBSP or proposed heuristics; (ii) the application runs with MigBSP, which performs the heuristics calculus and message-passing, but does not migrate any processes actually; (iii) the application runs with MigBSP and an heuristic to select the candidate processes for migration, enabling then any migration if it was evaluated as viable. The main idea is to show the overhead impact of the heuristic execution (comparison between scenarios (i) and (ii)) and performance impact when enabling migrations (comparison between scenarios (i) and (iii)).

Regarding the BSP application, we developed an implementation of the Lattice-Boltzmann method [29] to compute fluid dynamics. Technically, this method considers a typical volume of fluids composed of a collection of particles, where a particle is represented by a distribution function for each fluid component at each grid point. The data volume is divided into continuous blocks of equal size in accordance with the number of processes. Each block is copied and runs in a BSP process. After the computation phase, each process sends data to its right-sided neighbor. Finally, a synchronization barrier takes place and other superstep is computed afterwards.

5. Discussion of Results. The results consider the performance of MigCube and MigHull heuristics in terms of application processing time in Subsections 5.1 and 5.2. In addition, we also present two subsections, 5.3 and 5.4, for comparison purposes; the first one analyzes MigCube against MigHull and the second one compares both approaches with the standard process selection heuristics from MigBSP. We are using a BSP implementation of the fluid dynamics application with variations in the following configurations: number of supersteps; initial process-processor scheduling; the MigBSP's parameter denoted $\alpha$; and the aforementioned evaluation scenarios.

5.1. MigCube Evaluation. Table 5.1 shows the test results with MigCube. Scenario (ii) always produces a time larger than scenario (i), since the first adds the heuristic calculus and message passing. This overhead can be considered as part of the heuristic execution cost. The mean overhead of MigCube is $3.21 \%$. This overhead also takes place when migration are enabled but any process replacement is viable during the application execution. The effectiveness of MigCube appears when comparing scenarios (iii) and (i). The larger the number of supersteps, the larger the gains with process migration. In other words, an application that migrates the processes in the first supersteps presents better performance because of both it has more time to amortize the penalties involved in process migration and more time to execute with an optimized configuration. The highlighted fields show that only one migration happens when using 20 supersteps and $\alpha$ equal to 16 . Although achieving better results than scenario (i), the system remains unbalanced. This situation is only solved when 60 supersteps are performed.

Figure 5.1 shows the percentage of gain in execution time when analyzing scenarios (iii) and (i). It has been calculated using the following equation:

$$
\text { Gain }=\frac{\text { Scenario }(i)-\text { Scenario }(\text { iii })}{\text { Scenario }(i)} \times 100 .
$$

The parameter $\alpha$ equal to 8 was the responsible for the best results. A lower value of $\alpha$ implies in a greater number of process rescheduling calls (recalling that each call encompasses scheduling calculus and messagepassing), while a larger value of $\alpha$ parameter postpones the calls being so less reactive for process migration. When running a short number of supersteps, the configuration with $\alpha$ equal to 16 outperforms the other values 
Table 5.1: MigCube evaluation. The times are expressed in seconds. We are highlighting the execution of 20 supersteps with $\alpha=16$, where a single process migration takes place.

\begin{tabular}{|c|c|c|c|c|c|c|c|}
\hline \multirow{3}{*}{ Supersteps } & \multicolumn{7}{|c|}{ Scenarios } \\
\hline & \multirow{2}{*}{$\mathrm{i}$} & ii & iii & ii & iii & ii & iii \\
\hline & & \multicolumn{2}{|c|}{$\alpha=4$} & \multicolumn{2}{|c|}{$\alpha=8$} & \multicolumn{2}{|c|}{$\alpha=16$} \\
\hline \multicolumn{8}{|c|}{ Ascending } \\
\hline 20 & 16.10 & 17.39 & 11.79 & 16.57 & 10.85 & 16.33 & 14.33 \\
\hline 40 & 32.19 & 34.59 & 23.41 & 33.42 & 20.00 & 32.66 & 20.66 \\
\hline 60 & 48.28 & 51.86 & 36.29 & 49.93 & 28.32 & 48.99 & 27.42 \\
\hline 80 & 64.37 & 67.00 & 47.97 & 66.78 & 36.66 & 65.60 & 38.00 \\
\hline 100 & 80.47 & 84.90 & 60.85 & 83.28 & 46.20 & 81.88 & 44.61 \\
\hline \multicolumn{8}{|c|}{ Descending } \\
\hline 20 & 16.27 & 16.68 & 11.67 & 16.55 & 11.50 & 16.39 & 14.41 \\
\hline 40 & 32.94 & 33.09 & 21.47 & 33.28 & 21.36 & 33.20 & 21.99 \\
\hline 60 & 48.82 & 49.50 & 32.41 & 49.38 & 29.91 & 49.28 & 29.88 \\
\hline 80 & 65.09 & 65.80 & 42.21 & 65.78 & 38.58 & 65.63 & 40.82 \\
\hline 100 & 81.37 & 81.40 & 53.14 & 81.75 & 48.40 & 81.68 & 47.67 \\
\hline \multicolumn{8}{|c|}{$\mathrm{CPU}$} \\
\hline 20 & 20.08 & 20.33 & 13.78 & 20.30 & 16.97 & 20.13 & 19.50 \\
\hline 40 & 40.15 & 40.45 & 25.94 & 40.38 & 27.91 & 40.27 & 33.26 \\
\hline 60 & 60.22 & 60.56 & 39.16 & 60.50 & 38.28 & 60.47 & 41.28 \\
\hline 80 & 80.29 & 80.50 & 51.31 & 81.20 & 48.83 & 81.01 & 53.96 \\
\hline 100 & 100.36 & 100.87 & 64.50 & 100.63 & 60.27 & 100.51 & 62.12 \\
\hline \multicolumn{8}{|c|}{ Round-Robin } \\
\hline 20 & 16.16 & 17.11 & 10.73 & 16.42 & 10.95 & 16.30 & 14.28 \\
\hline 40 & 32.33 & 34.12 & 20.30 & 33.01 & 20.45 & 32.49 & 20.89 \\
\hline 60 & 48.46 & 51.13 & 30.94 & 49.29 & 29.38 & 48.68 & 27.73 \\
\hline 80 & 65.56 & 66.20 & 39.94 & 65.89 & 38.19 & 65.73 & 40.00 \\
\hline 100 & 80.66 & 83.50 & 49.99 & 82.17 & 48.00 & 81.20 & 47.09 \\
\hline
\end{tabular}

of $\alpha$ : with $\alpha=4$ or $\alpha=8$ we have a higher number of migrations (with time penalization on each migration activity) but not enough number of supersteps to amortize the investment in migrations. Figure 5.1 presents a linear behavior when considering the execution with $\alpha$ equal to 4 . In this case, process reorganization happens earlier and then, the execution can proceed with an optimized process-resource mapping after passing the first supersteps.

Figure 5.2 illustrates the number of migrations at each rescheduling call when considering $\alpha$ equal to 8 . Considering the CPU strategy for initial scheduling, we can observe that MigCube selects a large number of processes to migrate at each attempt. The $P M$ of the processes are closed to the largest $P M$, showing a large number of migrations at each rescheduling call. After analyzing the log of operations, we can observe a principle of hysteresis, i.e, several consecutive migrations in order to stabilize the behavior of the system. Moreover, in the current implementation, the migration test of a candidate process does not take into account the previous migration of other process to the same target (node or cluster), so contributing for the large number of observed migrations. These ideas explain the performance of the CPU strategy when compared to the remaining ones. Although obtaining good performance rates when comparing scenarios (i) and (iii), the CPU technique for initial scheduling achieves the worst performance among the other ones.

5.2. MigHull Evaluation. Table 5.2 shows the MigHull results. A mean overhead of $3.45 \%$ in the execution time was observed when comparing scenarios (i) and (ii). Considering scenario (iii), the same MigCube performance panorama appears here in MigHull, where larger gains appear when enlarging the number of supersteps. In particular, as presented in MigCube, the use of $\alpha$ equal to 16 was responsible for the best performance values when executing a short number of supersteps. Table 5.2 highlights the large difference in time when comparing the CPU initial mapping against the other three initial scheduling strategies. Although 

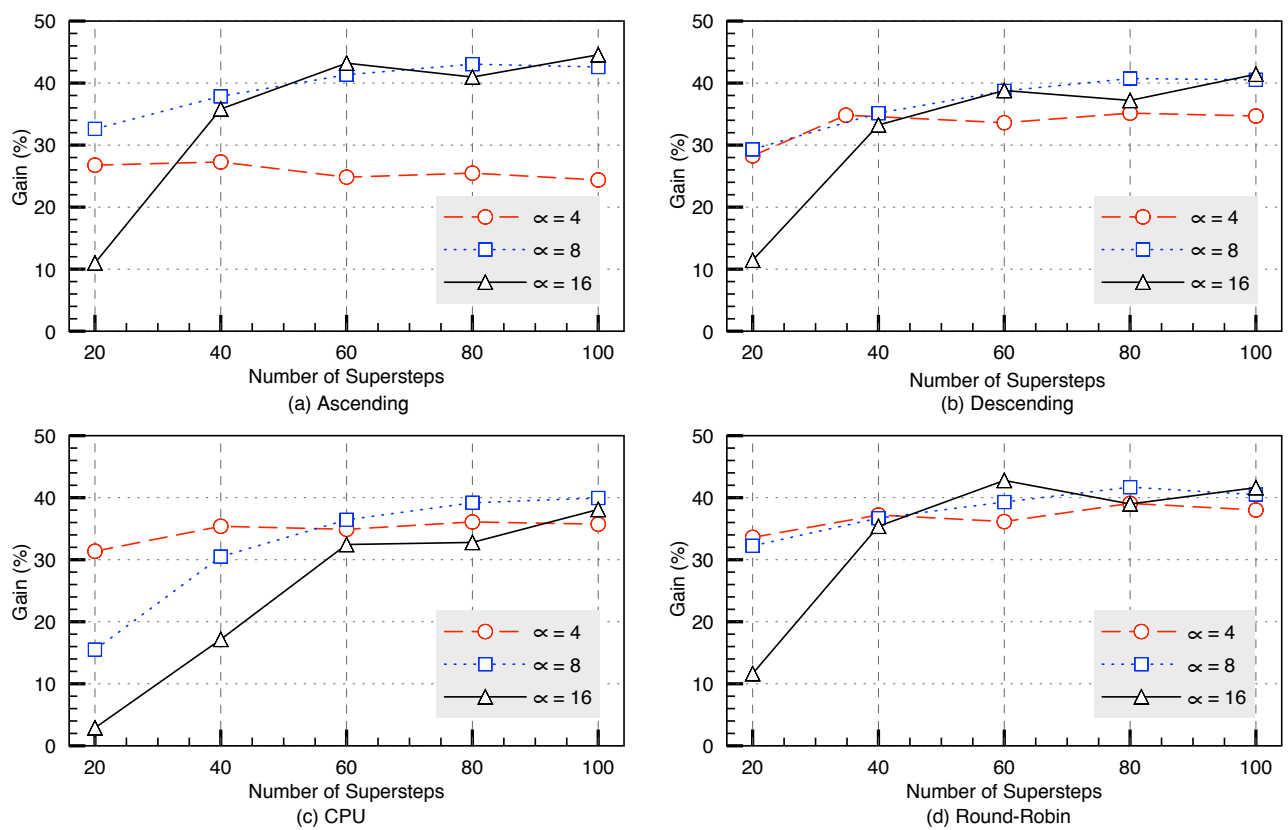

Fig. 5.1: Percentage of gain in the execution time with MigCube-driven process rescheduling

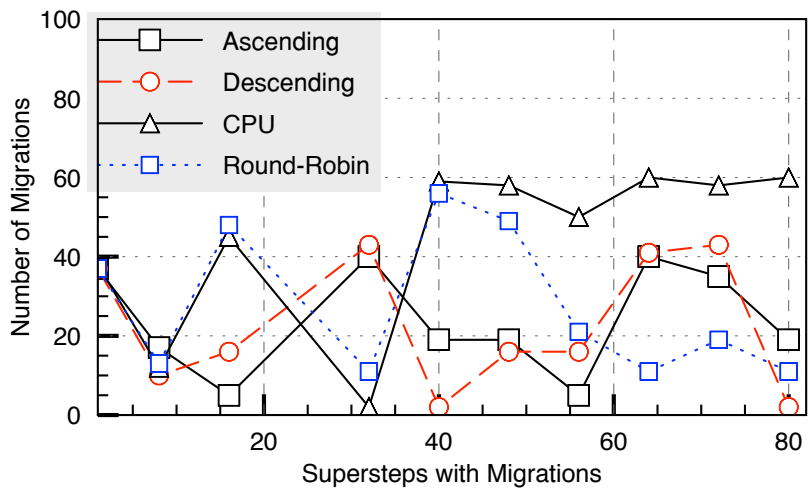

Fig. 5.2: Number of migrations at each rescheduling call when using MigCube and $\alpha=8$.

efficient in the CPU perspective, the CPU initial mapping causes communication penalties because there are a large number of inter-cluster communication.

Figure 5.3 shows the percentage of gain on the execution time when MigHull-driven migrations take place. The results were calculated considering Equation 5.1 and data from scenarios (i) and (iii). Analyzing Table 5.2 and the graphs in Figure 5.3 using the MigHull, it is possible to verify that a value of $\alpha$ equal to 8 is the most stable when considering the time gain. However, different from MigCube, a value of $\alpha$ equal to 16 does not show a tendency of gain in performance. The use of MigHull tends to be more complex and increases the cost of execution as increases the number of clusters, because each BSP process need to calculate the probability of migration in each cluster; in this way, increasing the computation cost. Figure 5.4 depicts the number of migrations at each rescheduling intervention when using $\alpha$ equal to 8 and the MigHull heuristic. Clearly, the 
Table 5.2: MigHull evaluation. The times are expressed in seconds. We are highlighting the performance of scenario (i), where the CPU strategy for initial mapping presents large disparities

\begin{tabular}{|c|c|c|c|c|c|c|c|}
\hline \multirow{3}{*}{ Supersteps } & \multicolumn{7}{|c|}{ Scenarios } \\
\hline & \multirow{2}{*}{$\mathrm{i}$} & ii & iii & ii & iii & ii & iii \\
\hline & & \multicolumn{2}{|c|}{$\alpha=4$} & \multicolumn{2}{|c|}{$\alpha=8$} & \multicolumn{2}{|c|}{$\alpha=16$} \\
\hline \multicolumn{8}{|c|}{ Ascending } \\
\hline 20 & 16.10 & 17.33 & 11.75 & 16.57 & 11.89 & 16.33 & 14.56 \\
\hline 40 & 32.19 & 34.59 & 21.52 & 33.42 & 21.95 & 32.66 & 23.04 \\
\hline 60 & 48.28 & 51.56 & 32.20 & 49.93 & 30.93 & 48.99 & 31.57 \\
\hline 80 & 64.37 & 67.04 & 42.07 & 66.78 & 39.91 & 64.60 & 42.39 \\
\hline 100 & 80.47 & 84.48 & 52.85 & 83.28 & 49.90 & 81.88 & 49.96 \\
\hline \multicolumn{8}{|c|}{ Descending } \\
\hline 20 & 16.27 & 16.68 & 12.15 & 16.60 & 11.99 & 16.39 & 14.31 \\
\hline 40 & 32.54 & 33.09 & 21.53 & 33.01 & 22.15 & 32.95 & 23.32 \\
\hline 60 & 48.82 & 49.50 & 32.27 & 49.38 & 31.12 & 49.25 & 32.12 \\
\hline 80 & 65.09 & 65.38 & 41.97 & 65.25 & 40.21 & 65.15 & 42.71 \\
\hline 100 & 81.37 & 81.70 & 53.11 & 81.65 & 50.22 & 81.53 & 51.37 \\
\hline \multicolumn{8}{|c|}{$\mathrm{CPU}$} \\
\hline 20 & 20.08 & 20.33 & 14.47 & 20.31 & 15.72 & 20.13 & 17.46 \\
\hline 40 & 40.15 & 40.45 & 27.04 & 40.37 & 27.05 & 40.29 & 28.46 \\
\hline 60 & 60.22 & 60.56 & 38.87 & 60.51 & 37.07 & 60.49 & 40.91 \\
\hline 80 & 80.29 & 81.10 & 51.84 & 81.02 & 47.37 & 80.95 & 55.03 \\
\hline 100 & 100.36 & 101.13 & 63.67 & 100.94 & 56.57 & 100.77 & 67.51 \\
\hline \multicolumn{8}{|c|}{ Round-Robin } \\
\hline 20 & 16.16 & 17.11 & 11.61 & 16.42 & 11.81 & 16.30 & 14.50 \\
\hline 40 & 32.33 & 34.12 & 21.20 & 33.01 & 21.93 & 32.49 & 23.04 \\
\hline 60 & 48.46 & 51.13 & 31.85 & 49.29 & 30.87 & 48.68 & 31.97 \\
\hline 80 & 64.56 & 65.15 & 41.44 & 65.89 & 39.87 & 65.09 & 43.20 \\
\hline 100 & 80.60 & 83.60 & 52.10 & 82.17 & 49.85 & 81.20 & 50.70 \\
\hline
\end{tabular}

MigHull strategy of using an intersection of the three 2D planes is responsible for reducing the number of migratable processes when compared to MigCube. Particularly, Figure 5.5 illustrates three moments of the execution for the Ascending strategy, showing the division of the processes among the clusters. We can observe the movement of the processes to take profit from the most powerful clusters, Chicon and Suno (see Section 4 for details regarding the subset of the Grid5000 infrastructure used in the tests).

5.3. MigCube and MigHull Comparative. Both MigCube and MigHull heuristics have the same objective and make use of the same idea: computational geometry to select a portion of process to migrate. Figure 5.6 illustrates the gains considering each initial scheduling and heuristic. The graph shows the mean value of gain of scenario (iii) over scenario (i) when considering all set of supersteps and $\alpha$ values. MigCube with the Ascending scheduling and $\alpha$ value equal to 4 achieved a gain of $25 \%$, diverging significantly from the other results. This divergence occurs due to a low $\alpha$, which makes many processes to migrate, increasing the communication between process and approximating metrics.

MigHull achieved up to $35 \%$ of gain in application execution time with process migration, while MigCube obtained $42 \%$. Figure 5.7 presents an analysis of the execution time of the supersteps at each migration call. The time presented in the graph refers to the interval between two supersteps in which a migration call took place. Particularly, we are considering 60 processes, 80 supersteps, $\alpha$ equal to 8 and the initial scheduling as being Round-Robin. In this way, migrations are evaluated at supersteps $1,8,16,24,32,40,48,56,64,72$ and 80. In this figure, the label 'Without Migration' represents the execution of the application without any migration, so the time is stable since the application performs the same number of computation activities at each superstep. We can observe that the time on both MigCube and MigHull tends to stabilize after crossing the 

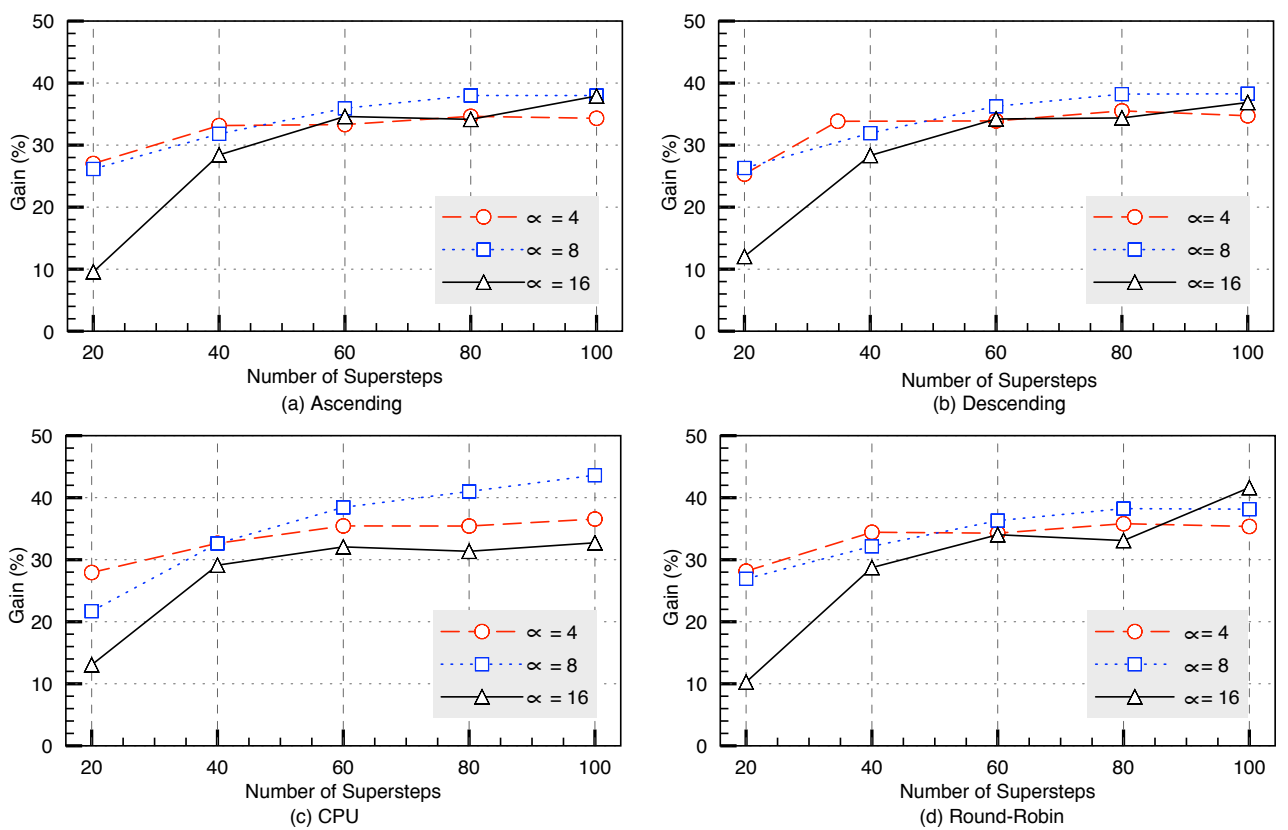

Fig. 5.3: Percentage of gain in the execution time with MigHull-driven process rescheduling

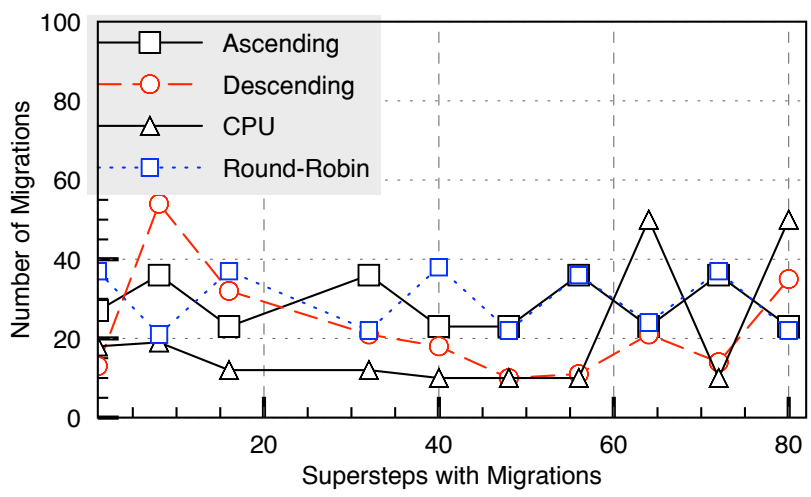

Fig. 5.4: Number of migrations at each rescheduling call when using MigHull and $\alpha=8$.

fourth migration call. Furthermore, we can observe that they achieved the main idea with process migration: to reduce the time of a superstep, so minimizing the application time as a whole.

5.4. Comparing MigCube and MigHull Against the Original Heuristics of MigBSP. Here, we intend to compare the proposed heuristics with the original ones, all developed for the scope of MigBSP. Up to the moment of MigCube and MigHull proposals, MigBSP offers two heuristics to select the candidate processes for migration, both of them based on the descending-sorted list of the highest PM of each process: (i) we can select the top of the list or; (ii) use a percentage to select a number of processes based on the value belonging to the top. While the first is not reactive, the second needs the user intervention to set a particular percentage for the application and execution environment duet. This last task is not trivial, mainly when dealing with heterogeneous and/or dynamic applications or parallel machines. Concerning this panorama, MigCube and 

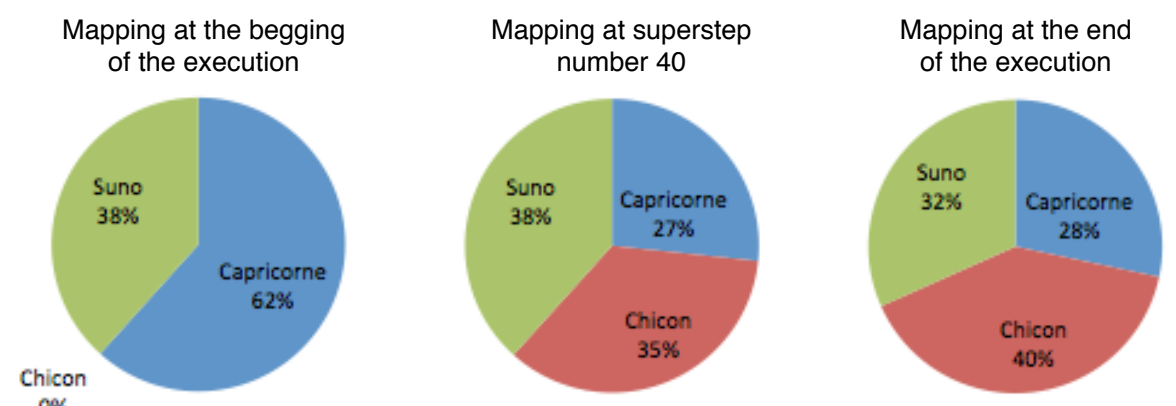

Fig. 5.5: Different moments of processes-clusters mappings when executing MigCube with the Ascending strategy for initial scheduling, 80 supersteps and $\alpha=8$.

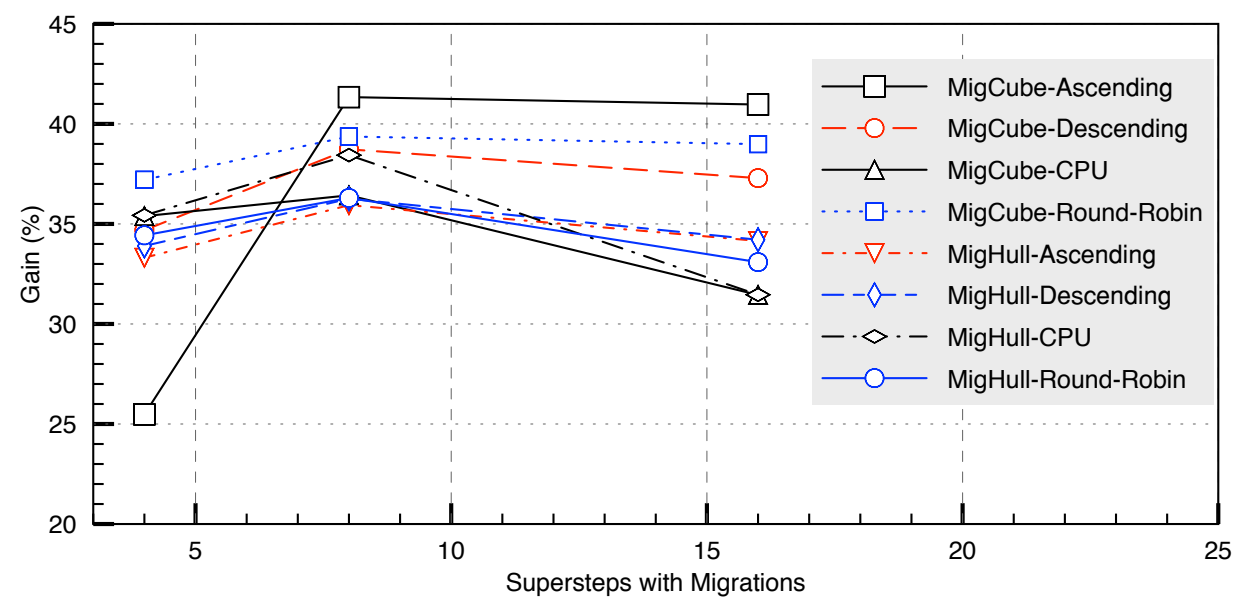

Fig. 5.6: Comparative involving MigCube and MigHull when varying the value of $\alpha$

MigHull come to fill the gap on process selection re-activity, not needing any intervention from the user nor previous knowledge about the BSP application.

Figure 5.8 shows a performance graph when considering the four aforementioned heuristics. This graph depicts, for each value of $\alpha$, a mean value of the executions with the four initial scheduling. The gain refers to the performance of scenarios (i) and (iii). As expected, the heuristic that selects only one process obtained the worst results. The heuristic of percentage selection, that is using a $20 \%$ selection from the top PM has similar results to MigCube and MigHull. This happens because the percentage heuristic can select more process at each execution, providing a fast rescheduling of processes. The MigCube and MigHull achieve the best results due its analysis of each metric and the use of geometrical space.

6. Related Work. Today, BSP represents the most used programming model to write successful parallel programs that exhibit phase-based computational behaviors. Thus, despite being proposed more than two decades ago by Leslie Valiant [33], several initiatives offer this model together with load balancing techniques and/or to treat particular parallel platforms [1, 8, 7, 37, 19, 21, 24, 27]. HAMA [1] is a cluster-driven library, particularly suitable for heterogeneous systems. It runs on top of the HDFS (Hadoop Distributed File System) in order to integrate BSP and iterative Map-Reduce applications. PUB [8, 7] is a C library that offers both 


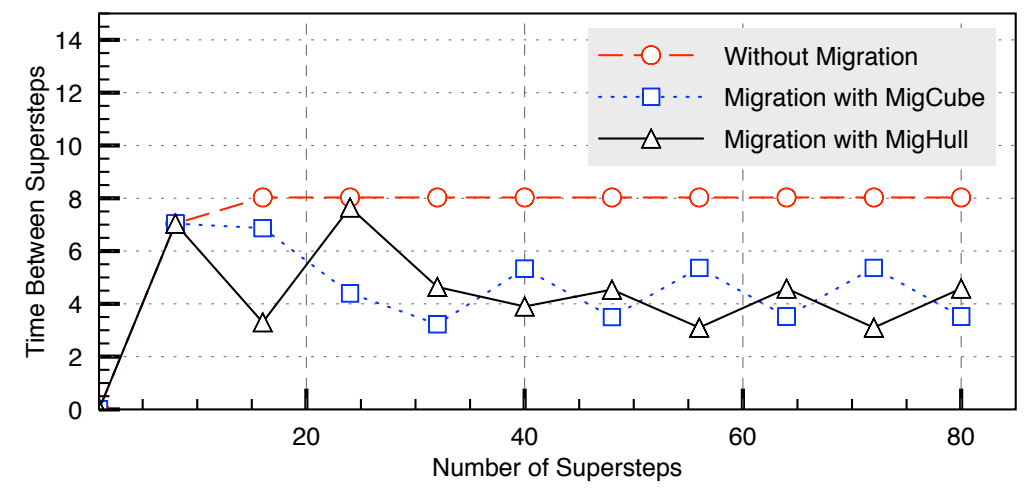

Fig. 5.7: Time between two supersteps in which migration calls took place.

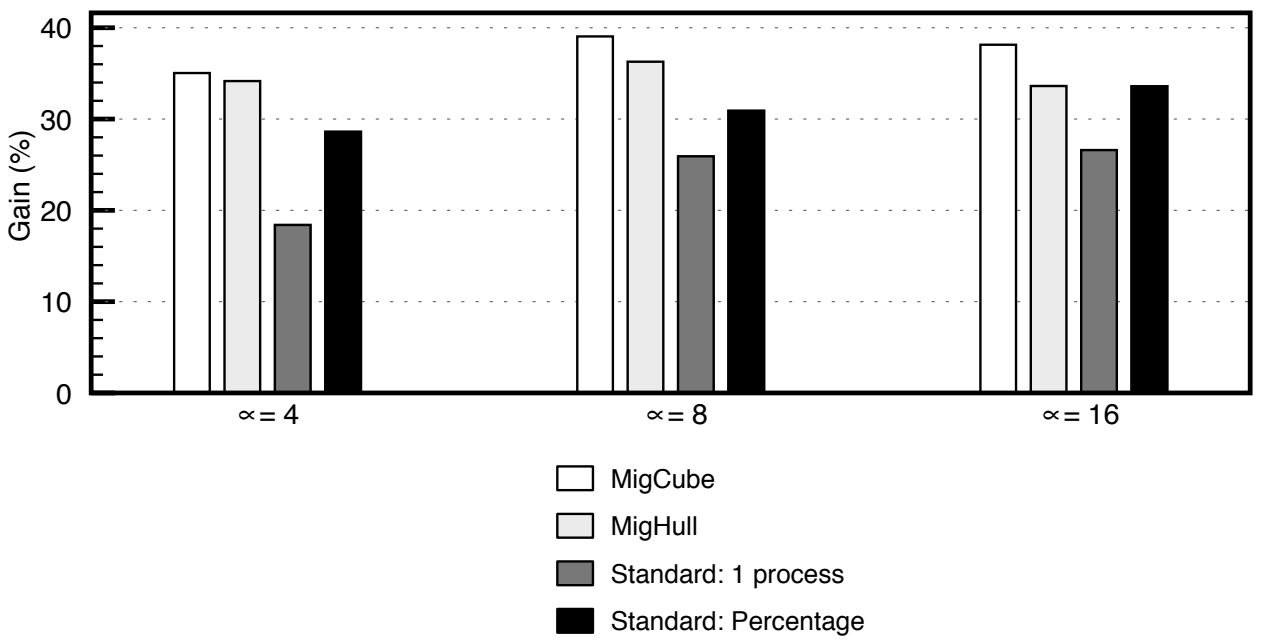

Fig. 5.8: Comparing MigCube and MigHull with the standard MigBSP (approaches to select the migratable processes: only the process in the top of the PM list and a percentage of processes based on the top value of this list).

centralized and distributed strategies for load balancing. In the first one, all nodes send data about their CPU power and load to a master node. The master verifies the least and the most loaded node and migrates one process between them. In distributed approaches, every node chooses $c$ (PUB parameter) other nodes randomly and asks them for their load. One process is migrated if the minimum load of $c$ analyzed nodes is smaller than own load of the node that is performing the test.

Mizan [19] monitors run-time characteristics of all processes (i.e., their execution time and incoming and outgoing messages). Using these measurements, at the end of every superstep, Mizan constructs a migration plan that minimizes the variations across workers by identifying which vertices to migrate and where to migrate them. BSPCloud [21] can make full use of multi-core clusters and has the advantage of performance predictability. Its target architecture are clusters, which are offered by cloud computing virtual machines. Pregel.NET [27] is based on Google's Pregel [23], offering distributed graph programming on the Azure Cloud using Bulk Synchronous Parallel model. It works with partitioning and scheduling of activities to workers in a Cloud environment, making use of the elasticity of virtual machines. Mansouri et al. [24] proposed task migration of a DSP (Digital 
Table 6.1: Related work comparison: F1 - Changing the application code; F2 - Platform: Grid or Cluster; F3 - Automatic selection of migratable processes, i.e., without user intervention; F4 - Use of computation metric (CPU load, CPU time or processing time) for load balancing; F5 - Use of communication data for load balancing; F6 - Use of migration costs for load balancing; F7 - Combination of metrics for load balancing purposes; F8 Support for BSP applications; F9 - Support of any kind of adaptivity on dynamic environments; F10 - Support for heterogeneous systems; F11 - Process migration capability

\begin{tabular}{|c|c|c|c|c|c|c|c|c|c|c|c|}
\hline \multirow{2}{*}{ Proposal } & \multicolumn{11}{|c|}{ Features } \\
\hline & F1 & $\mathrm{F} 2$ & F3 & $\mathrm{F} 4$ & $\mathrm{~F} 5$ & F6 & F7 & F8 & F9 & F10 & F11 \\
\hline MigBSP [28] & No & $\overline{\text { All }}$ & $\mathrm{No} \bullet$ & Yes & Yes & $\overline{Y e s}$ & Yes & Yes & Yes & Yes & Yes \\
\hline HAMA [1] & HDFS & Cluster & No & No & No & No & No & Yes & No & Yes & Yes \\
\hline PUB [7] & No & All & Yes & No & No & No & Yes & Yes & Yes & Yes & Yes \\
\hline MulticoreBSP [37] & Yes & No & $\mathrm{NA} \dagger$ & No & No & $\mathrm{NA} \dagger$ & No & Yes & Not & Not & No \\
\hline Mizan [19] & No & Cluster & Yes & No & No & No & Yes & Yes & Yes & Yes & Yes \\
\hline BSPCloud [21] & No & Cluster & No & No & No & No & Yes & Yes & Yes & No & Yes \\
\hline DistPM [20] & No & All & Yes & No & No & No & Yes & No & Yes & Yes & Yes \\
\hline Pregel.NET [27] & Yes & Cloud & Yes & No & No & No & No & Yes & Yes & Yes & Yes \\
\hline CPU-GPU cluster [24] & Yes & Cluster & Yes & No & No & No & No & Yes & Yes & Yes & Yes \\
\hline
\end{tabular}

Signal Processing) application implemented with the BSP computing model on a CPU-GPU cluster. During the processing phase of a BSP superstep, instead of moving the heavily loaded processes to another CPU, part of the load is divided to run in different GPUs. In this way, this middleware avoids network interaction, saving time on such operation. Unlike distributed systems, MulticoreBSP [37] library targets shared-memory computing employing thread-based parallelization. Finally, DistPM [20] is a library particularly developed to support process migration in grid computing. DistPM manages the network communication to avoid high data interaction between different clusters.

Table 6.1 summarizes the analysis of the aforementioned systems. We observe that our previous work named MigBSP is competitive among the BSP libraries regarding the load balancing perspective. Only MigBSP combines computation, communication and migration costs metrics for migration decision-making. Although having a process running in a slow processor that has a communication consistent pattern with a specific cluster, the migration penalties can act against migration viability, being dependent of process' size and network characteristics. The MigBSP's drawback considers how it selects the migratable processes, where now needs the intervention of user. In this way, both MigCube and MigHull proposed in this article seek to fill the MigBSP's gap, which is being used today to run BSP-based weather forecast and oil prospection applications in the south of Brazil [30].

7. Conclusion. Considering that the bulk synchronous style is a common organization on writing successful parallel programs [7, 10, 17], MigCube and MigHull emerge as alternatives for selecting their processes for running on more suitable resources without interference from the users. The key contribution of the proposed heuristics is the efficient use of computation, communication and migration costs metrics as axes values in the computational geometry for process migration decision-making. As mentioned above, MigCube and MigHull are not restricted to the MigBSP's scope, being employed to manage both heterogeneity and dynamism with process migration effortlessly at middleware level. Many data analysis techniques, such as machine learning and graph algorithms, require iterative computations and this is where Bulk Synchronous Parallel model can be more effective than MapReduce or Divide-and-Conquer strategies. The results showed gains larger than $40 \%$ when using MigCube or MigHull to decide process rescheduling in a subset of the Grid5000 environment. In addition, we also demonstrated a mean overhead close to $3 \%$ when employing the heuristics, but not performing any migrations. The evaluation emphasized the capacity of both proposed heuristics with different initial processes-processors mappings over an heterogeneous cluster-based grid.

Thus, future work includes the use of dynamism at resource and network usage levels to analyze MigCube and MigHull reactivity and overhead. The Lattice-Boltzmann application was very useful to present the benefits of the aforementioned heuristics in front of the originals presented in MigBSP, but we plan to evaluate the new 
proposals on new complex applications including weather prediction and DNA sequencing [30]. Moreover, the use of a simulator was very convenient to evaluate the MigCube and MigHull feasibility. In this way, also as future work, we are analyzing communication libraries such as ProActive ${ }^{3}$ and $\mathrm{AMPI}^{4}$ to implement MigBSP and the proposed heuristics. Consequently, real tests in the Grid5000 infrastructure will be conducted and compared with data obtained at simulation level.

Acknowledgements. This work was partially supported by the following Brazilian Agencies: CAPES, FAPERGS and CNPq.

\section{REFERENCES}

[1] Hama, June 2013. Available at: http://hama.apache.org/. Access: Jun. 2013.

[2] Simgrid, June 2013. Available at: http://simgrid.gforge.inria.fr/. Access: Jun. 2013.

[3] V. Arabnejad, A. Moeini, and N. Moghadam, Using bee colony optimization to solve the task scheduling problem in homogenous systems, International Journal of Computer Science Issues, 8 (2011), pp. 348-353.

[4] S. Bandyapadhyay, S. Bhowmick, and K. Varadarajan, Approximation schemes for partitioning: Convex decomposition and surface approximation, in Proceedings of the Twenty-Sixth Annual ACM-SIAM Symposium on Discrete Algorithms, SODA '15, SIAM, 2015, pp. 1457-1470.

[5] C. B. Barber, D. P. Dobkin, and H. HuhdanpaA, The quickhull algorithm for convex hulls, ACM Trans. Math. Softw., 22 (1996), pp. 469-483.

[6] M. D. Berg, O. Cheong, M. v. Kreveld, and M. Overmars, Computational Geometry: Algorithms and Applications, Springer-Verlag TELOS, Santa Clara, CA, USA, 3rd ed., 2008.

[7] O. Bonorden, Load Balancing in the Bulk-Synchronous-Parallel Setting using Process Migrations, 2007 IEEE International Parallel and Distributed Processing Symposium, (2007), pp. 1-9.

[8] O. Bonorden, B. Juurlink, I. von Otte, And I. Rieping, The paderborn university bsp (pub) library, Parallel Comput., 29 (2003), pp. 187-207.

[9] T. D. Braun, H. J. Siegel, N. Beck, L. L. Bölöni, M. Maheswaran, A. I. Reuther, J. P. Robertson, M. D. Theys, B. Yao, D. Hensgen, And R. F. Freund, A comparison of eleven static heuristics for mapping a class of independent tasks onto heterogeneous distributed computing systems, J. Parallel Distrib. Comput., 61 (2001), pp. 810-837.

[10] R. E. De Grande And A. Boukerche, Dynamic balancing of communication and computation load for hla-based simulations on large-scale distributed systems, J. Parallel Distrib. Comput., 71 (2011), pp. 40-52.

[11] G. El Kabbany, N. Wanas, N. Hegazi, and S. Shaheen, A dynamic load balancing framework for real-time applications in message passing systems, International Journal of Parallel Programming, 39 (2011), pp. 143-182.

[12] R. Fabbri, L. D. F. Costa, J. C. Torelli, and O. M. Bruno, 2d euclidean distance transform algorithms: A comparative survey, ACM Comput. Surv., 40 (2008), pp. 2:1-2:44.

[13] Z. Fan, H. Shen, Y. Wu, and Y. Li, Simulated-annealing load balancing for resource allocation in cloud environments, in Proceedings of the 14th International Conference on Parallel and Distributed Computing, Applications and Technologies (PDCAT'13), New York, NY, USA, 2013, IEEE, pp. 1-6.

[14] V. Gaba AND A. Prashar, Comparison of processor scheduling algorithms using genetic approach, International Journal of Advanced Research in Computer Science and Software Engineering, 2 (2012), pp. 37-45.

[15] M. R. Garey and D. S. Johnson, Computers and Intractability; A Guide to the Theory of NP-Completeness, W. H. Freeman \& Co., New York, NY, USA, 1990.

[16] S. Hashemi And A. Hanani, Solving the scheduling problem in computational grid using artificial bee colony algorithm, Advances in Computer Science: an International Journal, 2 (2013), pp. 37-41.

[17] B. Hendrickson, Computational science: Emerging opportunities and challenges, Journal of Physics: Conference Series, 180 (2009), p. 012013.

[18] S. Kardani-Moghaddam, F. Khodadadi, R. Entezari-Maleki, and A. Movagha, A hybrid genetic algorithm and variable neighborhood search for task scheduling problem in grid environment, Procedia Engineering, 29 (2012), pp. 3808-3814.

[19] Z. Khayyat, K. Awara, A. Alonazi, H. Jamjoom, D. Williams, and P. Kalnis, Mizan: a system for dynamic load balancing in large-scale graph processing, in Proceedings of the 8th ACM European Conference on Computer Systems, EuroSys '13, New York, NY, USA, 2013, ACM, pp. 169-182.

[20] Y. Li and Z. LAN, A novel workload migration scheme for heterogeneous distributed computing, in Cluster Computing and the Grid, 2005. CCGrid 2005. IEEE International Symposium on, vol. 2, 2005, pp. 1055-1062.

[21] X. Liu, W. Tong, And Y. Hou, BSPCloud: A Programming Model for Cloud Computing, 2012 IEEE 12th International Conference on Computer and Information Technology, (2012), pp. 1109-1113.

[22] A. Madureira, F. Santos, and I. Pereira, Self-managing agents for dynamic scheduling in manufacturing, in GECCO '08: Proceedings of the 2008 GECCO conference companion on Genetic and evolutionary computation, New York, NY, USA, 2008, ACM, pp. 2187-2192.

[23] G. Malewicz, M. Austern, And A. Bik, Pregel: a system for large-scale graph processing, Proceedings of the 2010 ACM SIGMOD International Conference on Management of Data, (2010), pp. 135-145.

\footnotetext{
${ }^{3}$ http://proactive.activeeon.com

${ }^{4}$ http://charm.cs.illinois.edu/research/ampi
} 
[24] F. Mansouri, S. Huet, V. Fristot, And D. Houzet, Task migration of DSP application specified with a DFG and implemented with the BSP computing model on a CPU-GPU cluster, Proccedings of the 2013 Conf. on Design and Architectures for Signal and Image Processing (DASIP), IEEE, 2013, pp. 326-333.

[25] M. F. PACE, BSP vs mapreduce, Procedia Computer Science, 9 (2012), pp. 246 - 255. Proceedings of the International Conference on Computational Science, ICCS 2012.

[26] J. PECERO AND P. BouvRY, An improved genetic algorithm for efficient scheduling on distributed memory parallel systems, in Proceedings of the International Conference on Computer Systems and Applications (AICCSA), New York, NY, USA, 2010, IEEE, pp. 1-8.

[27] M. Redekopp, Y. Simmhan, and V. Prasanna, Optimizations and analysis of bsp graph processing models on public clouds, in Parallel Distributed Processing (IPDPS), 2013 IEEE 27th International Symposium on, 2013, pp. $203-214$.

[28] R. D. R. Righi, L. Graebin, And C. A. DA Costa, On the replacement of objects from round-based applications over heterogeneous environments, Software: Practice and Experience, (2015), v. 45, n. 5, pp. 633-656.

[29] C. Schepke And N. Maillard, Performance improvement of the parallel lattice boltzmann method through blocked data distributions, in Computer Architecture and High Performance Computing, 2007. SBAC-PAD 2007. 19th International Symposium on, Oct 2007, pp. 71-78.

[30] J. Schneider, J. Gehr, H.-U. Heiss, T. Ferreto, C. De Rose, R. Righi, E. Rodrigues, N. Maillard, and P. Navaux, Design of a grid workflow for a climate application, in Computers and Communications, 2009. ISCC 2009. IEEE Symposium on, pp. 793-799.

[31] E.-G. TALBI, Metaheuristics : from design to implementation, John Wiley \& Sons, Inc., Hoboken, NJ, USA, 2009.

[32] H. Tang, Y. Zhou, X. Huang, And G. Rong, Does pareto's law apply to evidence distribution in software engineering? an initial report, in Proceedings of the 3rd International Workshop on Evidential Assessment of Software Technologies, EAST 2014, New York, NY, USA, 2014, ACM, pp. 9-16.

[33] L. G. Valiant, A bridging model for parallel computation, Commun. ACM, 33 (1990), pp. 103-111.

[34] H. Wu AND C. NIE, An overview of search based combinatorial testing, in Proceedings of the 7 th International Workshop on Search-Based Software Testing, SBST 2014, New York, NY, USA, 2014, ACM, pp. 27-30.

[35] A. Yzelman, R. Bisseling, D. Roose, And K. Meerbergen, Multicorebsp for c: A high-performance library for sharedmemory parallel programming, International Journal of Parallel Programming, (2013), pp. 1-24.

[36] K. Ponnavaikko and J. Dharanipragada, Wide Area Distributed Filesystems - A Scalability and Performance Survey, Scalable Computing: Practice and Experience (SCPE), v.11, n.3, (2010), pp. 305-325.

[37] A. Yzelman and R. H. Bisseling, An object-oriented bulk synchronous parallel library for multicore programming, Concurrency and Computation: Practice and Experience, 24 (2012), pp. 533-553.

[38] E. Cesario And D. Talia, Using Grids for Exploiting the Abundance of Data in Science, Scalable Computing: Practice and Experience (SCPE), v.11, n.3, (2010), pp. 251-261.

Edited by: Pedro Valero Lara

Received: Sept 9, 2015

Accepted: March 2, 2016 